ist auch ähnliches Festhaften der festen starken Base wie $\mathrm{NaOH}$ bzw. KOH an der Glaswand beim Lösen mit destilliertem Wasser zu erwähnen. Die starke AggTomeration der freien Kat- und Anionenzu erwahnen. Die starke Agglomeration der freien Kat- und Anionenaustauscherkörnchen (Mischbettbildung) könnte auch einen Schlüssel T. YAMABE: Bull. Chem. Soc. Japan 34, 1002 (1961).

\section{Zur Konstitution des Fragilins}

Vor kurzem haben wir über die Isolierung eines neuen Phenolglykosids (Fragilin) aus der Rinde von Salix fragilis $\mathbf{L}$. berichtet, das als Monoacetylderivat des Salicins erkannt wer den konnte1a). Die Stellung des Acylrestes an der Glucosemolekel blieb zunächst ungeklärt. Sie läßt sich — wie von uns auch am Beispiel des Salireposids gezeigt werden konnte $\left.{ }^{1 b}\right)-$ durch vollständige Methylierung des Glykosids, anschließende SpaItung und Identifizierung der gebildeten Methylglucose bestimmen. Da die bisher in Salix- und Populus-Arten gefundenen acylierten Glykoside den Säurerest entweder in 2- oder 6-Stellung der Glucosemolekel tragen, ist mit dem Auftreten von 3, 4, 6- bzw. 2, 3, 4-Trimethylglucose zu rechnen. Nach Untersuchungen von PEARL und DARLING ${ }^{2}$ ) ist bei den in 2-Stellung der Glucose acylierten Glykosiden bereits in schwach alkalischem Milieu eine Wanderung des Acylrestes in die 6-Stellung zu beobachten. Es muBte daher zunächst geprüft werden, ob im vorliegenden Fall unter den Bedingungen der Methylierung eine solche Umlagerung stattfindet. Dazu wurde eine Lösung von $20 \mathrm{mg}$ Fragilin in $1 \mathrm{ml}$ Dimethylformamid mit $50 \mathrm{mg}$ Silberoxid 12 Std geschüttelt. Aus dem Ansatz konnte das Glykosid unverändert wiedergewonnen werden; eine Acylwanderung konnte nicht beobachtet werden. Durch Magnesiumoxid wird Fragilin in wäBriger Lösung rasch in Salicin und Essigsäure gespalten.

Zur Bestimmung der Stellung des Acctylrestes wurde das Glykosid mit Methyljodid und Silberoxid in Dimethylformamid als Lösungsmittel permethyliert ${ }^{3}$ ). Der Ansatz wurde in wäßrig-methanolischer Lösung durch zweistündiges Kochen mit $5 \%$ iger Schwefelsäure hydrolysiert, anschließend auf dem Wasserbad vom Methanol befreit und mit Bariumcarbonat neutralisiert. Die erhaltene Lösung wurde nach dem Zentrifugieren papierchromatographisch mit den Verteilungsmitteln n-Butanol/Äthanol/Wasser (5:1:4) und Wasser/n-Butanol/Tetrachlorkohlenstoff $(4: 4: 3)$ auf Schleicher $u$. Schüll $2043 \mathrm{~b}$ mgl. (aufsteigend) auf Methylzucker geprüft. Als Vergleichssub stanzen wurden verwendet: $2,3,4,6$-Tetramethylglucose, 2,3,4Trimethylglucose, 3,4,6-Trimethylglucose und Glucose. Die Sichtbarmachung erfolgte durch längeres Erhitzen der mit Anilinphthalat besprühten Chromatogramme im Trockenschrank auf $110^{\circ} \mathrm{C}$. Neben geringen Mengen 2,3,4,6-Tetramethylglucose konnte als Hauptprodukt der Spaltung des permethylierten Fragilins 2,3,4-Trimethylglucose nachgewiesen werden. Der Acetylrest ist demzufolge in 6-Stellung an die Glucosemolekel gebunden. Fragilin konnte damit als 6-Acetylderivat des Salicins charakterisiert werden.

\section{Leipzig, Pharmazentisches Institut der Universitat}

Eingegangen am 28. Februar 1964

H. THIEME

1) Threme, H.: Naturwissenschaften a) 50, 477 (1963); b) 51, 291 (1964). - 2) Pearl, I.A., u. S. F. Darling: Arch. Biochem. Biophys. 102, 33 (1963). -3) Kunn, R., H. Trischmann u. I. Löw: Angew. Chem. 67, 32 (1955).

\section{Quantitative Bestimmungen der einzelnen Kollagenfraktionen} der Haut nach Anwendung von Resochin und Progesteron

Durch quantitative Bestimmung der Kollagen-Fraktionen sowie durch Untersuchung des Kollagen-Stoffwechsels mit Hilfe von ${ }^{14} \mathrm{C}-\mathrm{Glycin}$ bei lathyritischen, und zwar mit $\beta$-Aminopropionnitril behandelten, sowie bei mit Prednison behandelten Ratten konnte folgendes festgestellt werden:

Durch $\beta$-Aminopropionnitril wird die Kollagen-Synthese beschleunigt und der Übergang vom löslichen in unlösliches Kollagen verzögert ${ }^{1}$ ). Hingegen wird umgekehrt durch Prednison die Kollagen-Synthese verzögert und der Übergang vom löslichen in unlösliches Kollagen beschleunigt ${ }^{2}$ ).

An dieser Stelle berichten wir über den Einfluß von „Resochin“ (B) sowie ,Progesteron“*) auf das quantitative Verhalten der einzelnen Kollagen-Fraktionen bei der Ratte.

Angesetzt wurde eine Versuchsreihe mit 22 etwa $80 \mathrm{~g}$ schweren, weiblichen Albino-Ratten derselben Haltung. Resochin-Diphosphat und Progesteron wurden entsprechend der Tabelle 1 zugeführt:
Tabelle 1. Dosierung der Zugaben

Die Zahl der Tiere betrug je 5, bei den Kontrollen 7

\begin{tabular}{l|c|c|c}
\hline \multirow{2}{*}{ Wirkstoff und Applikationsart } & \multirow{2}{*}{$\begin{array}{c}\text { Versuchs- } \\
\text { dauer }\end{array}$} & \multicolumn{2}{|c}{ Menge (mg) } \\
\cline { 3 - 4 } & & je Tag & gesamt \\
\hline Resochin-Diphosphat per os . & 14 Tage & 10 & 140 \\
Resochin-Diphosphat per os . & 14 Tage & 15 & 210 \\
Progesteron i. m. . . . . . & 14 Tage & 0,5 & 7 \\
Kontrolltiere. . . . . . . . & 14 Tage & - & -
\end{tabular}

Die angewandten chemischen Kollagen-Bestimmungsmethoden waren dieselben wie in unseren vorausgehenden Untersuchungen $\left.{ }^{1}\right),{ }^{2}$ )

Evgebnisse: Wie aus Tabelle 2 hervorgeht, wurden bei den Ratten der geschilderten Versuchsanordnung das neutralsalzlösliche und das säurelösliche Kollagen am stärksten durch

Tabelle 2. Kollagen und nichtkollagenes Eiweiß in Häuten von unbehandelten, mit Resochin ${ }^{\circledR}$ sowie mit Progesteron behandelten Ratten

\begin{tabular}{|c|c|c|c|c|c|}
\hline \multirow{2}{*}{$\begin{array}{l}\text { Ratten, } \\
\text { behandelt mit }\end{array}$} & $\begin{array}{c}\text { Nicht } \\
\text { kollagenes } \\
\text { Eiweiß }\end{array}$ & $\begin{array}{c}\text { Gesamt- } \\
\text { kolla- } \\
\left.\text { gen }^{b}\right)\end{array}$ & \multicolumn{3}{|c|}{$\begin{array}{l}\text { Kollagen (in mg/100 mg } \\
\text { Gesamtkollagen })^{\mathrm{c}} \text { ) }\end{array}$} \\
\hline & \multicolumn{2}{|c|}{$\begin{array}{c}\text { in } \mathrm{mg} / 100 \text { feuchter } \\
\text { Haut }\end{array}$} & $\begin{array}{c}\text { neutral- } \\
\text { salzlöslich }\end{array}$ & \begin{tabular}{|l|} 
säure- \\
löslich
\end{tabular} & $\begin{array}{l}\text { un- } \\
\text { löslich }\end{array}$ \\
\hline Unbehandelt. & & $17,2 \pm 2,2$ & & $5,2 \pm 1,2$ & \\
\hline Resochin- $10 \mathrm{mg}$ & $18,0 \pm 4,2$ & & $6,4 \pm 1,5$ & $7,8 \pm 3,1$ & \\
\hline Diphosphat $15 \mathrm{mg}$ & $17,4 \pm 3,6$ & $16,6 \pm 2,1$ & $7,0 \pm 1,4$ & $8,2 \pm 1,2$ & $82 \pm$ \\
\hline Progesteron $0,5 \mathrm{mg}$ & $17,1 \neq 2,8$ & $15,3 \pm 3,6$ & $10,5 \pm 2,0$ & $9,0 \pm 1,9$ & $74 \pm$ \\
\hline
\end{tabular}

a) Berechnet aus Gesamtstickstoff der Haut minus Kollagenstickstoff.

b) Berechnet aus Hydroxyprolingehalt der Haut mal 7,46.

c) Gesamtkollagen, berechnet aus dem Hydroxyprolingehalt der feuchten Haut.

Progesteron erhöht, wobei diese Erhöhung die von uns bereits auch schon früher beobachtete ${ }^{3}$ ) gleichläufige Steigerung der beiden Fraktionen durch Resochin ${ }^{B}$ offenbar noch übertrifft. Mithin lassen sich auf jeden Fall sowohl durch Resochin B als auch durch Progesteron deutliche Veränderungen einzelner Kollagen-Fraktionen bewirken, und zwar in dem Sinne, daß durch Resochin (B) wie durch Progesteron das Verhältnis des löslichen zum reifen Kollagen deutlich zu den Vorstufen hin verschoben wird. Die Aufklärung weiterer Einzelheiten dieser Verschiebungen durch radioaktiv markierte Substanzen wird angestrebt.

Die Arbeit wurde mit finanzieller Unterstuitzung der Deutschen Forschungsgemeinschaft durchgeführt, der hierfür auch an dieser Stelle gedankt sei.

Mainz, Hautklinik dev Universität (Divektor: Prof. Dr. G.W. Korting), und Heidelberg, Anorganisch-Chemisches Institut der Universität (Direhtor: Prof. Dr. U. HoFMann)

H. Holzmann, G.W. Korting, F. Hammerstein, K. H. Stecher, M. Durruti, P. Iwangoff und K. Kühn

\section{Eingegangen am 17. Februar 1964}

*) Verabfolgt als Proluton ${ }^{\circledR}$ (Schering AG).

1) Kühn, K., M. Durruti, P. Iwangoff, F. Hammerstein, K.H. Stecher, H. Holzmann u. G. W. Korting: Hoppe-Seyler's Z. physiol. Chem. (im Druck). - 2) KÜH, K., P. IwANGOFF, F. Hammerstein, K.H. Stecher, M. Durruti, H. Holzvann u. G.W. Korting: Hoppe-Seyler's Z. physiol. Chem. (im Druck). 3) Kühn, K., H. Holzmann u. G. W. Korting: Naturwissenschaften 49, 134 (1962).

\section{Über netre Anthrachinonglykoside aus der Faulbaumrinde}

In den methanolischen Auszügen von Cortex Frangulae (Rhamnus Frangula L.) und Cortex Cascarae sagradae (Rhamnus Purshiana DC. = amerikanischer Faulbaum) des Handels lassen sich dünnschichtchromatographisch auf Kieselgel- oder Polyamidplatten außer den bereits bekannten Verbindungen noch mindestens 6 weitere Anthrachinonverbindungen nachweisen ${ }^{1}$ ), die bisher noch nicht beschrieben wurden. Die Isolierung eines Teils davon gelang durch Chromatographie an Polyamidsäulen, die Identifizierung durch $\mathrm{CH}$-Analyse, Darstellung von Acetaten, Úberführung in Methylprodukte sowie durch den Schmelzpunkt-, UV- und IR-Vergleich mit anderweitig isolierten oder von uns synthetisierten Verbindungen. 